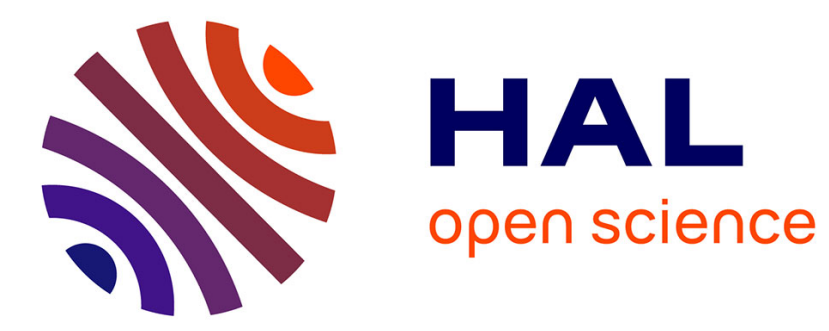

\title{
Evaluation de la longueur de diffusion par la méthode EBIC dans les piles solaires à l'AsGa et application à l'environnement spatial
}

\author{
J. Bozec, G. Rolland
}

\section{- To cite this version:}

J. Bozec, G. Rolland. Evaluation de la longueur de diffusion par la méthode EBIC dans les piles solaires à l'AsGa et application à l'environnement spatial. Revue de Physique Appliquée, 1986, 21 (8), pp.509-514. 10.1051/rphysap:01986002108050900 . jpa-00245468

HAL Id: jpa-00245468

https://hal.science/jpa-00245468

Submitted on 1 Jan 1986

HAL is a multi-disciplinary open access archive for the deposit and dissemination of scientific research documents, whether they are published or not. The documents may come from teaching and research institutions in France or abroad, or from public or private research centers.
L'archive ouverte pluridisciplinaire HAL, est destinée au dépôt et à la diffusion de documents scientifiques de niveau recherche, publiés ou non, émanant des établissements d'enseignement et de recherche français ou étrangers, des laboratoires publics ou privés. 


\title{
Evaluation de la longueur de diffusion par la méthode EBIC dans les piles solaires à l'AsGa et application à l'environnement spatial
}

\author{
J. Bozec et G. Rolland \\ CNES, Centre Spatial de Toulouse, 18, avenue Edouard Belin, 31055 Toulouse Cedex, France
}

(Reçu le 19 février 1985, révisé les 20 juin 1985 et 12 mars 1986, accepté le 25 avril 1986)

\begin{abstract}
Résumé. - Les performances électriques des cellules solaires dépendent en particulier de la longueur de diffusion. Ce paramètre physique donne une estimation de la qualité du matériau semiconducteur. Une des méthodes classiques de caractérisation de ce paramètre est la mesure du courant induit dans le composant par un faisceau d'électrons (EBIC). Cette méthode expérimentale ne donne pas directement la valeur réelle de la longueur de diffusion. Il est nécessaire de tenir compte de la vitesse de recombinaison en surface et des conditions de génération des porteurs dans le semiconducteur. Les résultats ont été comparés à ceux obtenus à partir de l'exploitation des courbes de réponse spectrale des cellules solaires. Nous analysons enfin, la dégradation de la longueur de diffusion en fonction de la dose de particules chargées simulant un environnement spatial.
\end{abstract}

\begin{abstract}
The electrical performances of components like solar cells depend partly on the diffusion length value. This physical parameter gives an estimation of the quality of the semiconductor. One well known method to characterize this parameter consists in an EBIC measurement but the experimental conditions usually do not give directly the actual value of the diffusion length. It is necessary to take into account both the surface recombination velocity and the carrier generation in the semiconductor. The results are compared with those obtained by fitting the spectral response of the solar cells. We analyse also the degradation on the diffusion length by the charged particules distributed near the earth in the magnetosphere.
\end{abstract}

\section{Introduction.}

La longueur de diffusion $(L)$ des porteurs minoritaires dans un semiconducteur est l'un des paramètres les plus importants permettant de contrôler la qualité du matériau semiconducteur dont dépendent les performances électriques des composants tels que les cellules solaires par exemple.

La méthode classique de mesure du courant induit (Fig. 1) par le faisceau d'électrons d'un microscope électronique à balayage peut être utilisée pour caractériser le paramètre $L$, mais les conditions expérimentales ne permettent pas d'accéder directement aux valeurs réelles de la longueur de diffusion. Il est nécessaire de tenir compte en particulier de la vitesse de recombinaison en surface et des conditions de génération dans le matériau.

A partir de mesures expérimentales, du courant induit dans la jonction d'une cellule solaire à l'arséniure de gallium, et d'un modèle théorique élaboré par Donolato [3], il a été possible d'apprécier la valeur de la longueur de diffusion des porteurs minoritaires.

Par la suite, les cellules solaires ont été soumises à un flux d'électrons de $1 \mathrm{MeV}$ simulant l'action des radiations ionisantes sur le générateur solaire d'un satellite en orbite géostationnaire. Une courbe longueur de diffusion, dose d'irradiation a pu être tracée.

\section{Aspect théorique.}

Vu la structure géométrique des composants que nous avons utilisés, le modèle théorique le mieux adapté décrivant les variations du courant EBIC avec la distance $x_{0}$ du faisceau à la jonction et les paramètres physiques de la structure est celui mis au point par Roos et Luke (Réf. [1]). Ce modèle prend en compte l'effet du champ arrière lié à la transition " $\mathrm{N}^{+}, \mathrm{N}^{\prime \prime}$ entre la couche active épitaxiée et la couche tampon en introduisant dans ce plan une vitesse de recombinaison effective $S_{\mathrm{B}}$. La valeur de cette vitesse de recombinaison effective est liée aux paramètres physiques des matériaux de part et d'autre du plan de l'interface (voir Réf. [2]). Les calculs présentés par Roos et Luke sont valables pour une répartition spatiale arbitraire du coefficient de génération des paires électron-trou créées par l'absorption du faisceau. L'équation obtenue (Eq. (A.18), Réf. [1]) nécessite un traitement numérique relativement lourd. 


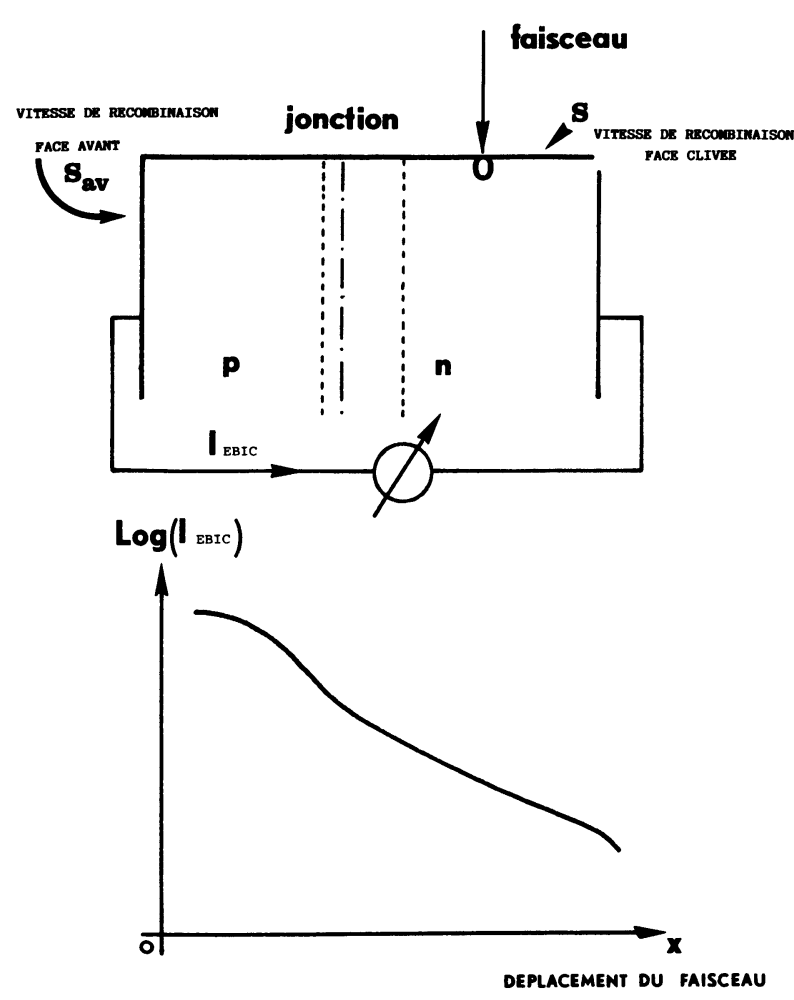

Fig. 1. - Schéma de principe.

[Schematic diagram.]

Cependant, nos courbes expérimentales gardant au

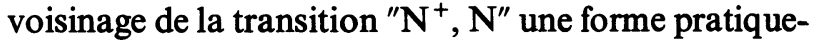
ment rectiligne, l'utilisation du modèle de Roos et Luke peut être évitée. En effet, dans ce cas particulier, la valeur de la vitesse de recombinaison en surface $S_{\mathrm{B}}$ est telle que l'action du champ à la transition $\mathrm{N}^{+}, \mathbf{N}$ compense l'effet lié à la présence du substrat à une distance finie de la jonction.

Cette remarque, jointe au fait que l'ordre de grandeur des valeurs de longueur de diffusion que l'on s'attend à trouver est bien inférieur à l'épaisseur de la couche $\mathrm{N}$, nous autorise à utiliser un modèle théorique plus simple correspondant à la situation décrite sur la figure 2. Le calcul de l'expression théorique du courant EBIC pour une telle structure, dans le cadre d'une répartition spatiale gaussienne du coefficient de génération, a été fait par Donolato (Eq. (30) de la Réf. [3]). On a :

$$
\begin{aligned}
I\left(X_{0}\right) & \sim \frac{2}{\pi} \int_{0}^{\infty} \frac{k}{\mu^{2}}\left[\exp \left(-\frac{k^{2} \sigma^{2}}{2}\right)-\frac{s}{\mu+s} \times\right. \\
& \left.\times \exp \left(\frac{\sigma^{2}}{2}-\mu Z\right) \cdot \frac{\operatorname{erfc}\left(\frac{\sigma \mu}{\sqrt{2}}-\frac{Z}{\sigma \sqrt{2}}\right)}{\operatorname{erfc}\left(-\frac{Z}{\sigma \sqrt{2}}\right)}\right]
\end{aligned}
$$$$
\times \sin \left(k X_{0}\right) \mathrm{d} k .
$$

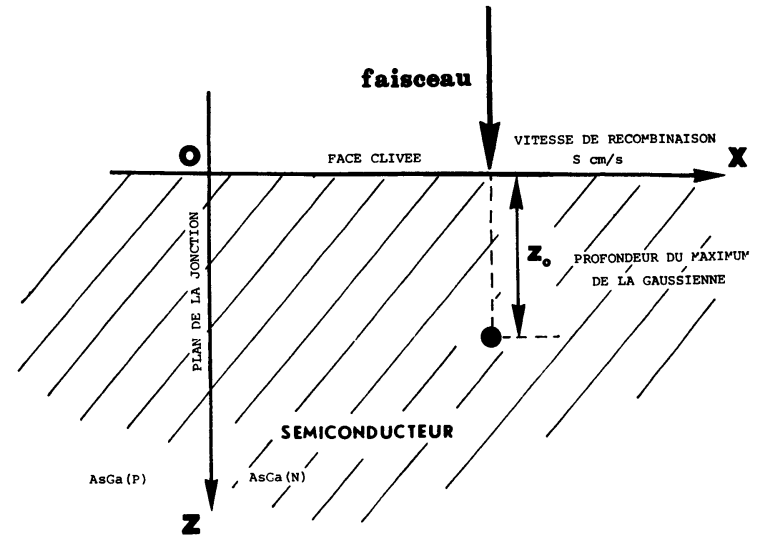

Fig. 2. - Conditions de l'expérience (génération non ponctuelle). L'axe des $Y$ est perpendiculaire à la figure.

[Experiment conditions (non punctual generation source). The $Y Y^{\prime}$ axis is perpendicular to the figure.]

Avec

$$
\left\{\begin{aligned}
\mu^{2} & =1+k^{2} \\
\sigma & =\frac{\sigma_{0}}{L} \\
s & =\frac{S L}{D} \\
Z & =\frac{z_{0}}{L} \\
X_{0} & =\frac{x_{0}}{L}
\end{aligned}\right.
$$

Les quantités $x_{0}, z_{0}, \sigma_{0}, S, L$ et $D$ sont respectivement : la position du faisceau sur l'axe des $x$ par rapport à la jonction; la profondeur du maximum de la gaussienne représentant le coefficient de génération; l'écart type de cette même gaussienne; la vitesse de recombinaison sur le plan d'entrée du faisceau; la longueur de diffusion et le coefficient de diffusion des minoritaires.

Les paramètres $z_{0}$ et $\sigma_{0}$ sont reliés à l'énergie du faisceau et aux caractéristiques du matériau par les relations :

$$
\begin{aligned}
& z_{0}=0,3 R \\
& \sigma_{0}=\frac{R}{\sqrt{15}}
\end{aligned}
$$

(Ref. [4] et Réf. [5]).

Le paramètre $R$ se déduit des formules de Kanaya (Réf. [4]); Pour l'AsGa :

$$
\begin{aligned}
R=1,72 & \times 10^{-2} \times \\
& \times E^{5 / 3} \frac{\left(1+9,78 \times 10^{-4} E\right)^{5 / 3}}{\left(1+1,957 \times 10^{-3} E\right)^{4 / 3}} \quad(\mu \mathrm{m}) .
\end{aligned}
$$


Où $E$ est l'énergie des électrons du faisceau exprimée en $\mathrm{keV}$.

Le calcul de l'intégrale décrivant le courant EBIC peut être réalisé facilement à partir de l'algorithme de Filon (Réf. [6], p. 890, no 25-4-54).

\section{Application à la mesure de $L$ dans des cellules solaires à l'AsGa.}

3.1 Elaboration DE LA CELlule SOlAIRE. - La cellule solaire élaborée est une homojonction p.n.n ${ }^{+}$ (Fig. 3) réalisée par les techniques d'élaboration de couches minces en épitaxie par CVD.OM. Les couches épitaxiées sont réalisées sur des substrats $10 \times 10 \mathrm{~mm}$ et d'épaisseur $350 \mu \mathrm{m}$, préalablement préparés par décapage chimique. Les épaisseurs des couches déposées et leurs concentrations en impuretés de dopage sont mesurées avec un détecteur de profil de concentration POLARON 4000.

L'épaisseur de la zone active a été volontairement augmentée pour répondre aux exigences de l'expérience.

Epaisseur de la couche $\gg L$.

Trois lots de piles solaires ont été préparés à différentes concentrations de porteurs majoritaires dans la zone active de type $\mathrm{n}$. Les conditions expérimentales d'élaboration assurent dans le réacteur d'épitaxie une très bonne homogénéité des substrats.

Les valeurs obtenues sur un échantillon témoin sont généralisées à l'ensemble du lot correspondant (Tableau I).

Ensuite, les substrats épitaxiés subissent pour une géométrie définie, les processus de photogravure par lift-off, de prise de contacts ohmiques et de dépôt de couches antireflet, caractéristiques de la technologie de fabrication des cellules solaires à l'arséniure de gallium.

Un certain nombre de paramètres physiques sont nécessaires à la détermination de la longueur de diffusion dans la méthode de calcul : les épaisseurs des couches, leurs concentrations en dopant et la mobilité des porteurs minoritaires.

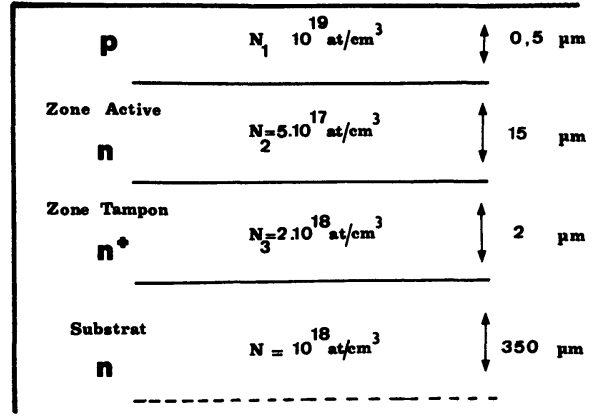

Fig. 3. - Exemple de dépôt réalisé par épitaxie.

[Example of epitaxial growth.]

Des mesures de mobilité de Hall, par la méthode de Van der Pauw, sont effectuées sur des échantillons témoins élaborés en même temps que les couches épitaxiées. Ces témoins permettent de déterminer ces différents paramètres.

3.2 DÉtermination DE LA MOBILITÉ DES PORTEURS MINORITAIRES. - La mobilité de Hall et la mobilité de conduction sont liées par le facteur de Hall, $r_{\mathbf{H}}$ :

$$
r_{\mathbf{H}}=\frac{\mu_{\mathrm{H}}}{\mu_{\mathrm{C}}}
$$

Dans l'AsGa à la température de $300 \mathrm{~K}, r_{\mathbf{H}}$ vaut 1,1 pour un échantillon de type $\mathrm{N}$ et 0,85 pour un échantillon de type $P$, références [7] et [8].

Les mobilités de conduction des électrons et des trous dans un semiconducteur sont liées par le rapport :

$$
\frac{\mu_{\mathrm{n}}}{\mu_{\mathrm{p}}}=b
$$

La figure 4 donne la variation du rapport $b$ en fonction de la concentration [9]. Cette valeur de $b$ nous permet d'approcher la valeur de la mobilité des porteurs minoritaires à partir des relations (5) et (6).

Tableau I. - Paramètres physiques des cellules solaires étudiées.

\begin{tabular}{|c|c|c|c|c|c|c|}
\hline Lot & $\begin{array}{c}N_{1} \\
\text { Zone p } \\
\mathrm{At} / \mathrm{cm}^{3}\end{array}$ & $\begin{array}{c}\mu_{1} \\
\mathrm{~cm}^{2} \cdot \mathrm{v}^{-1} \mathrm{~s}^{-1}\end{array}$ & $\begin{array}{c}N_{2} \\
\text { Zone n } \\
\text { At } / \mathrm{cm}^{3}\end{array}$ & $\stackrel{\mu_{2}}{\mathrm{~cm}^{2} \cdot \mathrm{v}^{-1} \mathrm{~s}^{-1}}$ & $\begin{array}{c}N_{3} \\
\text { Zone } \mathrm{n}^{+} \\
\text {et Substrat } \\
\mathrm{At} / \mathrm{cm}^{3}\end{array}$ & $\begin{array}{c}\mu_{3} \\
\mathrm{~cm}^{2} \cdot \mathrm{v}^{-1} \mathrm{~s}^{-1}\end{array}$ \\
\hline 1 & $4 \times 10^{18}$ & 330 & $2 \times 10^{17}$ & 388 & $1,3 \times 10^{18}$ & 393 \\
\hline 2 & $10^{19}$ & 94 & $5 \times 10^{17}$ & 363 & $2 \times 10^{18}$ & 454 \\
\hline 3 & $3 \times 10^{18}$ & 294 & $8 \times 10^{17}$ & 303 & $2 \times 10^{18}$ & 454 \\
\hline
\end{tabular}

[Physical parameters of the investigated solar cells.] 


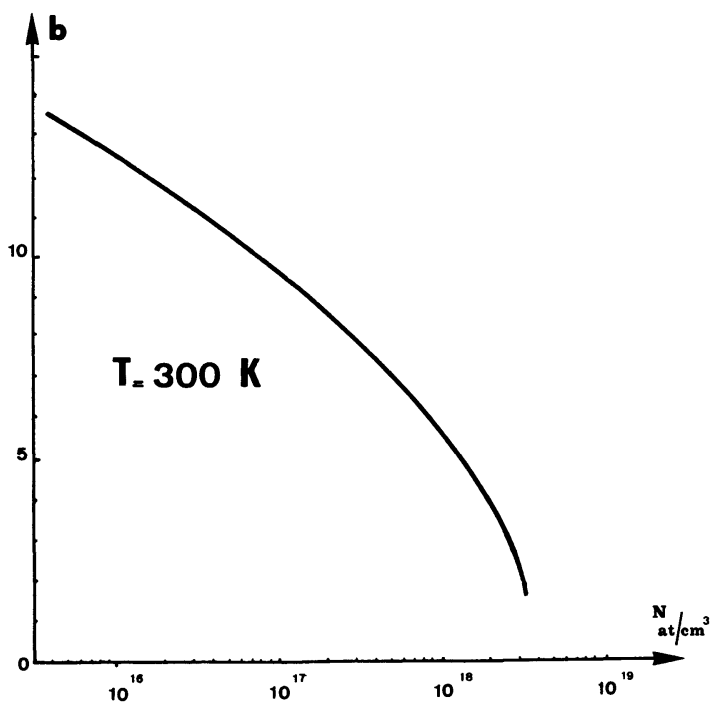

Fig. 4. - Variation de $b=\frac{\mu_{\mathrm{n}}}{\mu_{\mathrm{p}}}$ en fonction de la concentration en dopant.

[Variation of $b=\frac{\mu_{\mathrm{n}}}{\mu_{\mathrm{p}}}$ versus impurities concentration. $]$

3.3 MESURE DE LA LONGUEUR DE DIFFUSION PAR LA RÉPONSE SPECTRALE. - La réponse spectrale des piles est enregistrée sur un banc de mesures. Cette courbe est tracée point par point à différentes longueurs d'onde sélectionnées par des filtres interférentiels; on extrait ensuite de cette courbe, la courbe de rendement de collecte interne (voir Réf. [2]). La mesure de la puissance lumineuse incidente pour chaque longueur d'onde est effectuée par une cellule solaire AsGa étalon. La comparaison des courbes théoriques [10] et expérimentales de réponses spectrales des cellules solaires, à l'aide d'un programme de calcul, permet d'apprécier l'ordre de grandeur de la longueur de diffusion des porteurs minoritaires de la couche active et de confirmer les résultats de la méthode EBIC. Cette méthode de comparaison est soumise cependant à l'appréciation de deux paramètres difficiles à déterminer : la vitesse de recombinaison en face avant de la pile solaire et le coefficient de réflexion de la couche antireflet. La comparaison des résultats reste néanmoins intéressante (voir Tableau II).

3.4 Conditions eXPérimentales (EBIC). - L'expérience est montée à l'intérieur de la chambre de test d'un microscope électronique à balayage (JEOL 50A).

La cellule solaire à tester est clivée, afin de réduire les effets de surface, puis l'échantillon est monté sur un support adapté au porte échantillon du microscope. Ce support comprend également un cylindre de Faraday, pour mesurer le courant produit par le faisceau d'électrons incidents, et une mire d'étalonnage pour contrôler le grossissement du microscope.

Le faisceau d'électrons est déplacé sur la surface clivée de l'échantillon. La position du faisceau est repérée sur l'écran cathodique du M.E.B. Connaissant le gain du microscope, par le contrôle de la mire d'étalonnage, on peut mesurer les déplacements $x \mathrm{du}$ point d'impact. Pour chaque position du faisceau, on mesure le courant induit qui traverse la jonction et on trace la courbe $I(x)$.

La juxtaposition des courbes théoriques élaborées par un programme de calcul, et des courbes expérimentales permet de déterminer la valeur du paramètre longueur de diffusion (Fig. 5).

Nous avons pu également constater que la variation de l'énergie du faisceau entre 10 et $30 \mathrm{keV}$ ne modifiait pas les résultats obtenus de la valeur de la longueur de diffusion (Fig. 6). Les résultats portés dans le tableau II ont été obtenus à une valeur d'énergie de faisceau constante $(E=20 \mathrm{keV})$, assurant un compromis correct entre un courant induit élevé et un diamètre de la poire d'excitation petit devant l'épaisseur de la couche active.

\section{Application de la méthode EBIC à l'étude de la dégradation des piles solaires sous flux de particules chargées.}

L'étude de dégradation a été menée dans le cas d'un générateur solaire de satellite en orbite géostationnaire pour une durée de vie de 7 années. La simulation en

Tableau II. - Longueur de diffusion (en microns) mesurée à différentes doses en électrons de $1 \mathrm{MeV} / \mathrm{cm}^{2}$.

[Diffusion length measured in micrometers for different values of $1 \mathrm{MeV}$ electron fluence.]

\begin{tabular}{c|c|c|c|c|c|c|c|c}
\multirow{2}{*}{ Lot } & \multicolumn{2}{|c|}{ Dose 0 } & \multicolumn{2}{c|}{ Dose $3 \times 10^{13}$} & \multicolumn{2}{c|}{ Dose $3 \times 10^{14}$} & \multicolumn{2}{c}{ Dose $10^{15}$} \\
\cline { 2 - 9 } & EBIC & Rep. Spec. & EBIC & Rep. Spec. & EBIC & Rep. Spec. & EBIC & Rep. Spec \\
\hline 1 & 13,3 & 11,3 & 7,6 & 6 & 1,6 & 1,6 & 0,63 & 0,7 \\
\hline 2 & 8,1 & 6,2 & 5,8 & 4 & 1,22 & 1,1 & 0,62 & 0,6 \\
\hline 3 & 3 & 2,8 & 2,6 & 2,2 & 1,2 & 1,2 & 0,58 & 0,6 \\
\hline
\end{tabular}




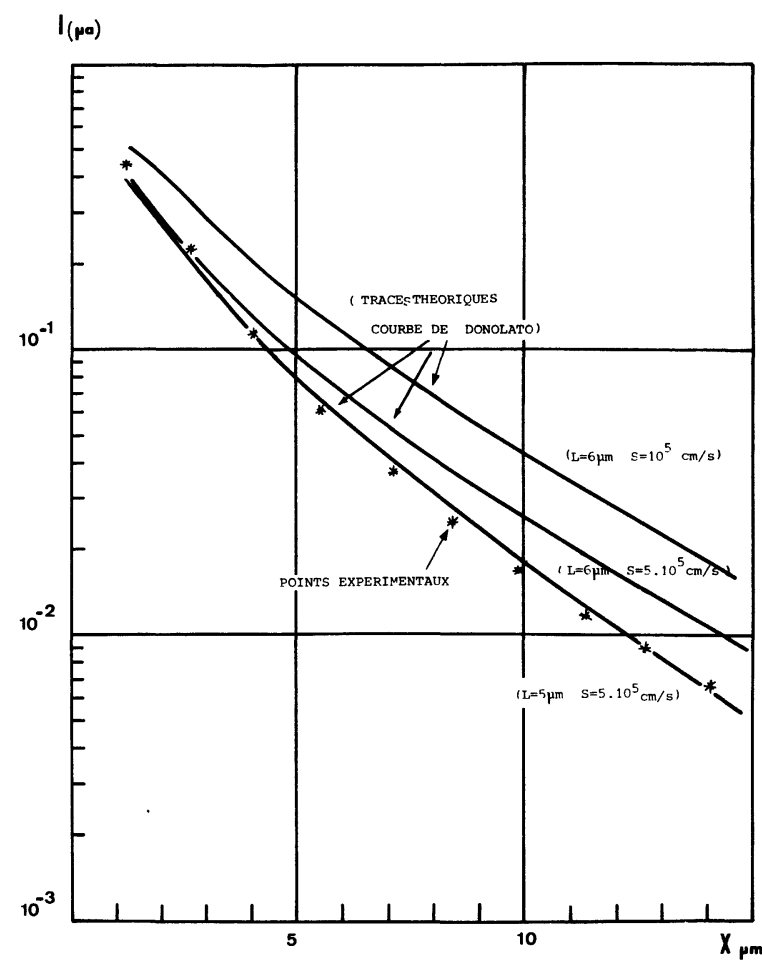

Fig. 5. - $I_{\text {EBIC }}$ : courbe expérimentale et courbes théoriques de Donolato. Dose reçue $3 \times 10^{13}$ electrons de $1 \mathrm{MeV} / \mathrm{cm}^{2}$. Energie du faisceau du MEB $=20 \mathrm{keV}$.

[ $I_{\text {EBIC }}$ : experimental curve and theoretical curve from Donolato. $\left(1 \mathrm{MeV}\right.$ Electron fluence $=3 \times 10^{13} \mathrm{e} / \mathrm{cm}^{2}$ SEM Beam Energy $=20 \mathrm{keV})$.]

laboratoire en dose équivalente d'électrons de $1 \mathrm{MeV}$ a pris en compte pour cette période l'ensemble du flux d'électrons reçus dans la zone de piégeage stable et du flux de protons d'éruptions solaires. La totalité de la dose équivalente d'électrons de $1 \mathrm{MeV}$ au bout de 7 années d'existence a été estimée à $N=10^{15}$ électrons $/ \mathrm{cm}^{2}$.

Les irradiations successives des piles solaires ont été réalisées avec un accélérateur Van der Graff dans une installation sous vide. Pour obtenir un faisceau d'électrons uniforme sur toute la surface du plateau qui reçoit les cellules, les particules sont diffusées à travers une mince feuille d'aluminium.

Le tableau II et les figures 7 et 8 rendent compte des résultats obtenus.

La méthode de mesure du courant induit permet d'accéder dans de bonnes conditions aux valeurs de la longueur de diffusion des porteurs minoritaires des couches actives de cellules solaires irradiées.

La figure 8 donne les valeurs de la longueur de diffusion $L$ en fonction de la dose en électrons de $1 \mathrm{MeV}$ reçue et pour différentes concentrations en porteurs majoritaires de la couche active. Les résultats obtenus sont semblables à ceux obtenus par d'autres méthodes, en particulier en D.L.T.S. [11] (Deep-Level Transient Spectroscopy).

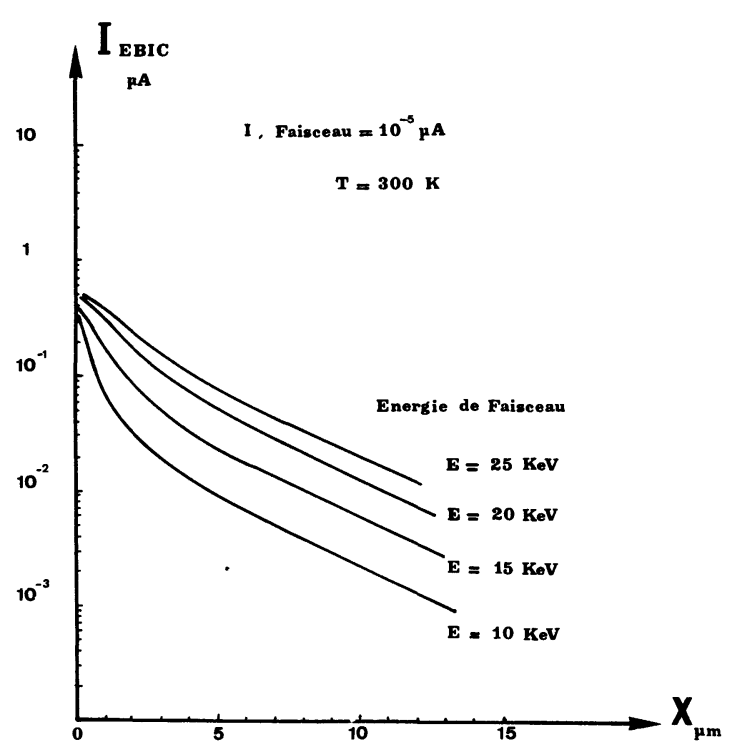

Fig. 6. $-I_{\text {EBIC }}$ : en fonction du déplacement du faisceau à différentes énergies de faisceau.

[ $I_{\text {EBIC }}$ as a function of the beam position for different values of beam accelerating voltage.]

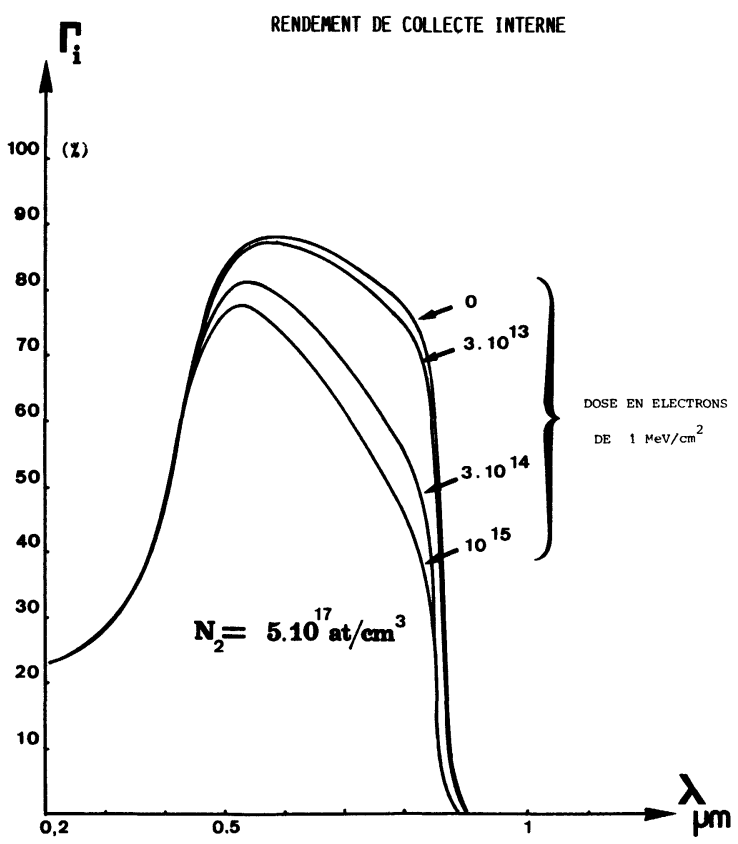

Fig. 7. - Dégradation du rendement de collecte interne en fonction de la dose en électrons de $1 \mathrm{MeV}$.

[Degradation of the spectral response as a function of $1 \mathrm{MeV}$ electron fluence.]

\section{Conclusion.}

Nous avons utilisé une méthode de mesure par courant induit (EBIC) pour évaluer la valeur réelle de la longueur de diffusion dans une structure photovol- 


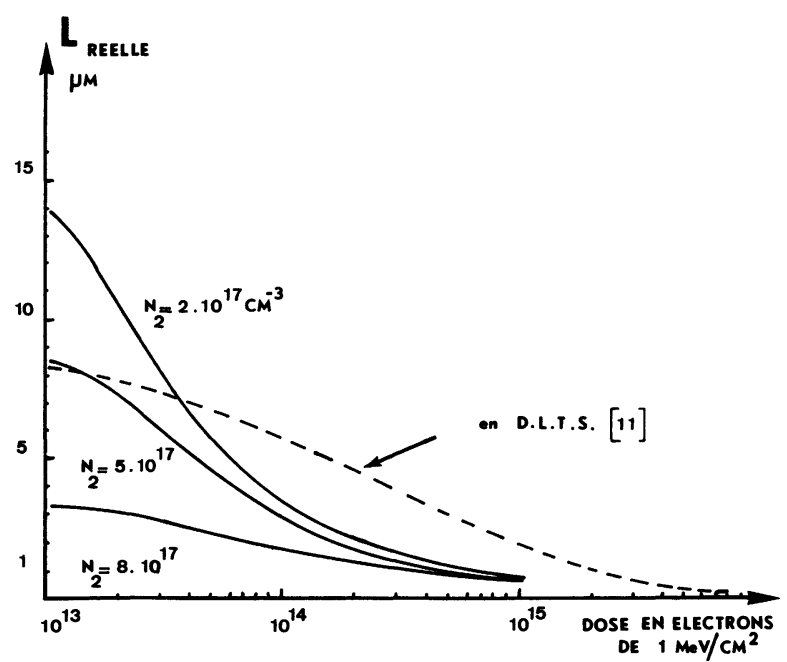

Fig. 8. - Variation de la longueur de diffusion réelle en fonction de la dose reçue.

[Diffusion length value as a function of $1 \mathrm{MeV}$ evaluation fluence.] taïque à l'arséniure de gallium dont la couche active est réalisée par épitaxie en CVD-OM. Le modèle théorique utilisé pour décrire le courant EBIC est celui proposé par Donolato (cf. § 2).

Les résultats obtenus sont voisins de ceux trouvés par d'autres méthodes (D.L.T.S.) ou (Réponse Spectrale).

Nous montrons, de plus, que la technique de croissance en phase vapeur aux organométalliques sous pression réduite permet d'obtenir des valeurs élevées de longueur de diffusion.

Les résultats de l'étude en irradiation, condensés sur les courbes de la figure 8 , peuvent servir de point de départ à des études de dégradations de piles solaires en ambiance spatiale.

\section{Bibliographie}

[1] Roos et LUKE, Analysis of the interaction of an electron beam with back surface field solar cells. J. Appl. Phys. 54 (7) (1983).

[2] Orgeret, M., Les piles Solaires, le composant et ses applications, Masson, 1985.

[3] Donolato, C., On the analysis of diffusion length Measurements by SEM. Solid State Electron. 25, $\mathrm{n}^{\circ} 11$ (1982).

[4] Kanaya and Sokayama, Penetration and energy-Loss theory of electrons in solid targets, J. Phys. D : Appl. Phys. 5 (1972).

[5] Fitting, GlaefeKe and Wild, Electron penetration and Energy Transfer in Solid Targets, Phys. Status Solidi (a) 43 (1977) 185.

[6] Abramowitz and STEGUn, Handbook of Mathematical functions (Dover Publications) 1970.
[7] Rode, D. L., Low Field Electron Transport, Semiconductors and semi-metals, Vol. 10 (Willardson).

[8] RoIzes, Communication personnelle, CERT Toulouse DERTS.

[9] Visentin, N., Contribution à la caractéristique de substrats semi-isolants d'arséniure de gallium. Thèse Université Paul Sabatier, Toulouse, 1979.

[10] Rolland, G., Rapport interne - CNES/DRT/QPE/ EP - 239 .

[11] LANG, W. L. and Sheng, S. H., Study of Deep Level Defects and Recombinaison Parameters One MeV electron and Low Energy Proton irradiated AlGaAs/GaAs Solar Cells, 17th IEEE Photovoltaïc Specialists Conference, Orlando F1, May 1.4. 1984. 